\title{
Chronic sciatic nerve compression induces fibrosis in dorsal root ganglia
}

\author{
QINWEN LI ${ }^{1 *}$, JIANGHAI CHEN $^{2 *}$, YANHUA CHEN $^{2}, \mathrm{XIAOBIN} \mathrm{CONG}^{2}$ and ZHENBING CHEN ${ }^{2}$ \\ ${ }^{1}$ Department of Orthopedics, The First People's Hospital of Yichang, Yichang, Hubei 443000; ${ }^{2}$ Department of Hand Surgery, \\ Union Hospital, Tongji Medical College, Huazhong University of Science and Technology, Wuhan, Hubei 430022, P.R. China
}

Received February 28, 2015; Accepted December 15, 2015

DOI: $10.3892 / \mathrm{mmr} .2016 .4810$

\begin{abstract}
In the present study, pathological alterations in neurons of the dorsal root ganglia (DRG) were investigated in a rat model of chronic sciatic nerve compression. The rat model of chronic sciatic nerve compression was established by placing a $1 \mathrm{~cm}$ Silastic tube around the right sciatic nerve. Histological examination was performed via Masson's trichrome staining. DRG injury was assessed using Fluoro Ruby (FR) or Fluoro Gold (FG). The expression levels of target genes were examined using reverse transcription-quantitative polymerase chain reaction, western blot and immunohistochemical analyses. At 3 weeks post-compression, collagen fiber accumulation was observed in the ipsilateral area and, at 8 weeks, excessive collagen formation with muscle atrophy was observed. The collagen volume fraction gradually and significantly increased following sciatic nerve compression. In the model rats, the numbers of FR-labeled DRG neurons were significantly higher, relative to the sham-operated group, however, the numbers of FG-labeled neurons were similar. In the ipsilateral DRG neurons of the model group, the levels of transforming growth factor- $\beta 1$ (TGF- $\beta 1$ ) and connective tissue growth factor (CTGF) were elevated and, surrounding the neurons, the levels of collagen type I were increased, compared with those in the contralateral DRG. In the ipsilateral DRG, chronic nerve compression was associated with significantly higher levels of phosphorylated (p)-extracellular signal-regulated kinase $1 / 2$, and significantly lower levels of p-c-Jun N-terminal kinase and p-p38, compared with those in the contralateral DRGs. Chronic sciatic nerve compression
\end{abstract}

Correspondence to: Dr Zhenbing Chen, Department of Hand Surgery, Union Hospital, Tongji Medical College, Huazhong University of Science and Technology, 1037 Luoyu Road, Wuhan, Hubei 430022, P.R. China

E-mail: qwli.2007@outlook.com; 2990430205@qq.com

*Contributed equally

Key words: chronic sciatic nerve compression, dorsal root ganglia, transforming growth factor- $\beta 1$, connective tissue growth factor, collagen type I, mitogen-activated protein kinase likely induced DRG pathology by upregulating the expression levels of TGF- $\beta 1$, CTGF and collagen type I, with involvement of the mitogen-activated protein kinase signaling pathway.

\section{Introduction}

Chronic peripheral nerve injury induces morphological and phenotypic alterations in nerve fibers, leading to hyperalgesia and allodynia $(1,2)$. It has been suggested that chronic nerve compression affects millions of individuals (3). Pathological changes have been found at the site of nerve compression after 1 month and, at 8 months, the damage has been observed to have progressed throughout the nerve (4). However, the precise mechanism underlying the pathogenesis of nerve compression injury remains to be fully elucidated. Studies have shown that chronic sciatic nerve compression triggers a series of pathological changes in the areas close by, including breakdown of the blood nerve barrier, sub-perineurial edema, fibrosis, and localized and diffuse demyelination (3,5-8). Details of the fibrotic process following nerve compression are unclear, and phenotypic changes in the neurons of the dorsal root ganglia (DRG) at the damaged site are accompanied by upregulated or downregulated gene and protein expression levels $(9,10)$. In addition, neuronal inflammation of the DRG as a response to chronic nerve compression has been noted (11).

Collagen fibers are the most important component of the extracellular matrix, and the excessive production of collagen type I may contribute to the pathogenesis of fibrosis (12). Transforming growth factor- $\beta$ (TGF- $\beta$ ) is reported to be prominent in sensory neurons, promote fibrosis associated with pathological pain (13) and induce the expression of connective tissue growth factor (CTGF) (14). It has been suggested that increased CTGF in the painful disc is associated with disc fibrosis and degeneration (15).

The mitogen-activated protein kinase (MAPK) signaling pathway has been found to be activated in DRG neurons during neuropathic pain in cell and rat models (16-18). However, the involvement of the collagen type 1, TGF- $\beta$, CTGF and MAPK signaling proteins in neuropathic pain remains to be fully elucidated. The present study hypothesized that these factors and mediators may support and contribute to the pathological changes that are associated with chronic sciatic nerve compression, particularly fibrosis, in DRG neurons and in the surrounding tissues. 
In the present study, a rat model of chronic sciatic nerve compression was established to investigate the subsequent signs of fibrosis, and the dynamics of the collagen type 1 , TGF- $\beta 1$, CTGF and MAPK family members in the DRG neurons and surrounding tissues. The results from the current study will provide further understanding of the pathology of chronic sciatic nerve compression-induced fibrosis in dorsal root ganglia.

\section{Materials and methods}

Reagents. Fluoro Ruby (FR) and Fluoro Gold (FG) were purchased from Molecular Probes (Thermo Fisher Scientific, Inc., Waltham, MA, USA) and Fluorochrome LLC (Denver, CO, USA), respectively. Silastic tubes were purchased from Shanghai Daoguan Rubber Products (Shanghai, China). Polymerase chain reaction (PCR) primers were obtained from Invitrogen (Thermo Fisher Scientific, Inc). Rabbit polyclonal anti-TGF- $\beta 1$ (cat. no. sc-146) and rabbit polyclonal anti-CTGF antibodies (cat. no. sc-25440) were obtained from Santa Cruz Biotechnology, Inc. (Santa Cruz, CA, USA). Rabbit monoclonal anti-glyceraldehyde 3-phosphate dehydrogenase (GAPDH; cat. no. 2251-1) and rabbit polyclonal anti-collagen I (cat. no. ab34710) antibodies were purchased from Epitomics, Inc. (Burlingham, CA, USA) and Abcam (Cambridge, UK), respectively. Rabbit monoclonal anti-phosphorylated (p-)ERK (cat. no. 4370) and rabbit monoclonal anti-p-p38 (cat. no. 4511) antibodies were purchased from Cell Signaling Technology, Inc. (Danvers, MA, USA). Rabbit polyclonal anti-p-c-Jun N-terminal kinase (JNK; ab4821) antibody was purchased from Abcam. Horseradish peroxidase (HRP)-conjugated goat anti-rabbit secondary antibody (cat. no. 074-1506) was purchased from KPL, Inc. (Gaithersburg, MD, USA). Secondary antibodies, including AlexaFluor 488-conjugated goat anti-rabbit IgG (cat. no. ANT030) and AlexaFluor 594-conjugated goat anti-rabbit IgG (cat. no. ANT051) antibodies, were purchased from Antgene Biotech (Wuhan, China).

Animals. A total of 100 male Sprague-Dawley rats (age, 7-8 weeks; weight, 250-300 g) were purchased from the Laboratory Animal Center, Tongji Medical College, Huazhong University of Science and Technology (Wuhan, China) and housed at $22^{\circ} \mathrm{C}$ with a $12 \mathrm{~h}$ light/dark cycle. Each animal had ad libitum access to food and water. All experimental procedures and protocols were approved by the Board of Ethics of Tongji Medical College, Huazhong University of Science and Technology.

Establishment of the chronic sciatic nerve compression model. The chronic sciatic nerve compression model was established in accordance with the method described by O'Brien et al (19). The animals were anesthetized using pentobarbital $(40 \mathrm{mg} / \mathrm{kg}$ intraperitoneal injection) and the right sciatic nerve of the rat was exposed through a gluteal muscle splitting approach. In the model rat group $(\mathrm{n}=8)$, a $1 \mathrm{~cm}$-long Silastic tube (internal diameter $1 \mathrm{~mm}$; outer diameter $2 \mathrm{~mm}$; Shanghai Daoguan Rubber Products Factory, Shanghai, China) was cut longitudinally, and placed atraumatically around the sciatic nerve. The longitudinal split in the tube was then closed using 8-0 sutures
(Shanghai Pudong Jinhuan Medical Product Co., Ltd., Shanghai, China). The left sciatic nerve was exposed, as above, but remained unbanded and was returned to its original position to serve as a comparative control. In the sham-operated group $(n=8)$, the right sciatic nerves were exposed, using the same procedure as described above for the left sciatic nerve of the model rats, but without Silastic tube placement.

Subsequently, 3 weeks following the above procedures, the ipsilateral and contralateral L4-L6 dorsal root ganglia (DRG) were harvested from the above experimental groups ( $n=8$ for each group). At 3 or 8 weeks after the establishment of the animal model, rats were sacrificed using an overdose of phenobarbital (Wuhan Boster Biological Technology, Ltd., Wuhan, China), and the ipsilateral and contralateral gastrocnemius muscles were collected from the above experimental groups ( $\mathrm{n}=8$ for each group).

Retrograde tracing using FR or FG. FR and FG are fluorescent tracers used for the retrograde labeling of afferent neurons in rodents (20). At 2 weeks post-surgery, the rats were anesthetized. The right sciatic nerves were exposed again, and the FR and FG fluorescent tracers were injected intraneurally. In the rats in the chronic sciatic nerve compression model, $5 \mu \mathrm{l} \mathrm{FR}$ (5\% dissolved in normal saline) was injected into the proximal end of the compressed nerve, and $5 \mu \mathrm{l} \mathrm{FG} \mathrm{(5 \%} \mathrm{dissolved} \mathrm{in}$ normal saline) was injected into the distal nerve. In the sham control rats, $5 \mu \mathrm{l} 5 \% \mathrm{FR}$ and $5 \mu \mathrm{l} 5 \% \mathrm{FG}$ were injected into the same respective sites as in the model rats. Following injection, the muscle and incision were closed.

Retrograde tracing analysis. At 1 week post-fluorescent tracer injection, the ipsilateral DRG of L4, L5 and L6 were removed and fixed in 4\% paraformaldehyde (Wuhan Boster Biological Technology, Ltd.). The DRGs were then dehydrated, embedded in optimal cutting temperature compound (Applygen Technologies Inc., Beijing, China) and sectioned coronally using a microtome (Leica CM1510S; Leica Microsystems, Wetzlar, Germany). The fluorescence was examined under a fluorescent microscope (Olympus IX71; Olympus Corporation, Tokyo Japan), and the numbers of FR- and FG-positive neurons were analyzed using ImagePro Plus version 6.0 (Media Cybernetics, Inc., Rockville, MD, USA).

Masson's trichrome staining of gastrocnemius muscle. The ipsilateral and contralateral gastrocnemius muscles were fixed and embedded in paraffin (Wuhan Boster Biological Technology, Ltd.). The gastrocnemius muscles were stained with Masson's trichrome (Harris hematoxylin, 1\% hydrochloric-alcohol solution, acid fuchsin, $1 \%$ phosphomolybdic acid and $2 \%$ aniline blue). The morphological details of Masson's trichrome staining were examined using a light microscope (CKX41; Olympus Corporation). A total of six images, each from the ipsilateral and contralateral gastrocnemius muscles, were randomly selected to measure the average muscle fiber cross-sectional area (CSA) and collagen volume fraction (CVF). The CVF was calculated as follows: CVF $(\%)=($ collagen area / total area) $\times 100$ in muscle fiber CSA.

Reverse transcription-quantitative PCR (RT-qPCR). At 3 weeks following the establishment of the chronic sciatic 
Table I. Sequences of forward and reverse primers used for reverse transcription-quantitative polymerase chain reaction analysis.

Target gene Primer sequence

$\begin{array}{ll}\beta \text {-actin } & \text { F: 5'-CGTTGACATCCGTAAAGACCTC-3' } \\ & \text { R: 5'-TAGGAGCCAGGGCAGTAATCT-3' } \\ \text { TGF- } \beta 1 & \text { F: 5'-TACTACGCCAAAGAAGTCACCC-3' } \\ & \text { R: 5'-TCCCGAATGTCTGACGTATTGA-3' } \\ \text { CTGF } & \text { F: 5'-CGGGAAATGCTGTGAGGAGT-3' } \\ & \text { R: 5'-CAGTTGGCTCGCATCATAGTT-3' } \\ \text { Collagen I } & \text { F: 5'-CCTACAGCACGCTTGTGGATG-3' } \\ & \text { R: 5'-AGATTGGGATGGAGGGAGTTTAC-3 }\end{array}$

TGF- $\beta 1$, transforming growth factor- $\beta 1$; CTGF, connective tissue growth factor; F, forward; R, reverse.

nerve compression model, the contralateral and ipsilateral L4-L6 DRG tissues were removed. Total RNA was extracted from the homogenized tissue samples using TRIzol reagent, chloroform and isopropyl alcohol (Wuhan Boster Biological Technology, Ltd.). After centrifugation at 7,500 x g at $4^{\circ} \mathrm{C}$ for 5 min, samples were collected and dried at room temperature. The total RNA was resolved in RNA-free water. The samples were reverse-transcribed using a First Strand cDNA Synthesis kit (Toboyo Co., Ltd., Osaka, Japan). The RT-qPCR was performed using a sequence detection system (StepOne Real-Time PCR System; Thermo Fisher Scientific, Inc.). PCR amplification was performed using Thunderbird SYBR qPCR mix (12.5 $\mu$; Toboyo Co., Ltd.) with $2 \mu \mathrm{l}$ specific primers $(2.5 \mu \mathrm{M}$; Table I), $2 \mu \mathrm{l}$ cDNA and $8.5 \mu \mathrm{l}$ double distilled water. The PCR reactions were as follows: Pre-denaturation at $95^{\circ} \mathrm{C}$ for $1 \mathrm{~min}$, denaturation at $95^{\circ} \mathrm{C}$ for $30 \mathrm{sec}$, annealing at $58^{\circ} \mathrm{C}$ for $30 \mathrm{sec}$ and extension at $72^{\circ} \mathrm{C}$ for $20 \mathrm{sec}$ (for a total of 40 cycles). $\beta$-actin was used as the internal control. The relative expression levels from the amplified RNA samples were calculated using the $2^{-\Delta \Delta \mathrm{Cq}}$ method (21).

Immunostaining. At 3 weeks following the establishment of the chronic sciatic nerve compression model, the contralateral and ipsilateral L4-L6 DRG tissues were removed, embedded in paraffin and sectioned. The samples were blocked with normal goat serum (Wuhan Boster Biological Technology, Ltd.) and probed with anti-TGF- $\beta 1$ (1:50 dilution), anti-CTGF (1:50 dilution) or anti-collagen I (1:200 dilution) at $4^{\circ} \mathrm{C}$ overnight. The following day, the slides were washed three times with phosphate-buffered saline and incubated with secondary antibody at $4^{\circ} \mathrm{C}$ overnight, then washed with Tris-buffered saline plus Tween-20 (TBS-T; $0.1 \%$ Tween-20). Following washing, the nuclei were counterstained with DAPI $(100 \mu \mathrm{g} / \mathrm{ml}$; Wuhan Boster Biological Technology, Ltd.), and samples were mounted in antifade reagents (Beyotime Institute of Biotechnology, Haimen, China). The fluorescence was examined under a fluorescent microscope (Olympus IX71; Olympus Corporation).
A A 0 weeks 3 weeks 8 weeks

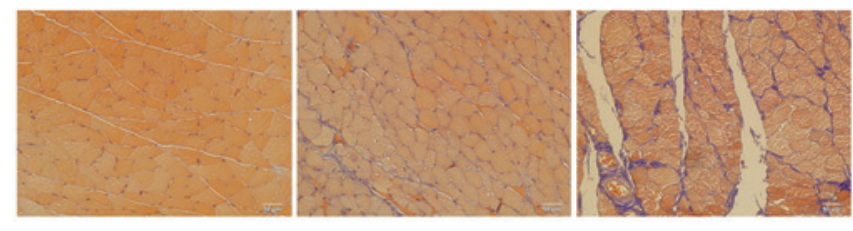

B

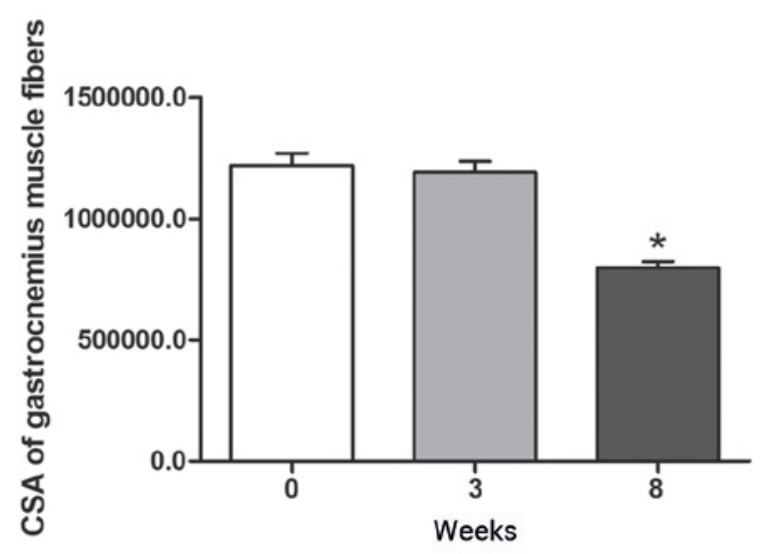

$\mathbf{C}$

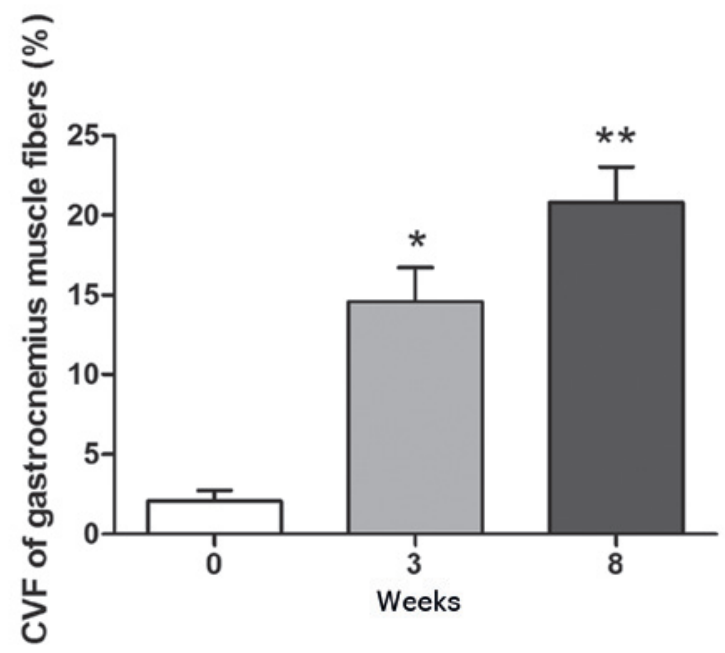

Figure 1. Muscle fiber changes in rats with chronic sciatic nerve compression. The rats were subjected to sciatic nerve compression for 0,3 or 8 weeks. (A) Muscles stained with Masson's trichrome. Representative images show collagen fiber and muscle formation. Scale bar $=50 \mu \mathrm{m}$. (B) Gastrocnemius muscle fiber CSA in different groups. (C) Percentage of gastrocnemius muscle fiber CVF in different groups. Data are presented as the mean \pm standard deviation. ${ }^{*} \mathrm{P}<0.05$ and ${ }^{* *} \mathrm{P}<0.01$ compared with the control ( 0 weeks). CSA, cross-sectional area; CVF, collagen volume fraction.

Protein extraction and western blot analysis. At 3 weeks following establishment of the chronic sciatic nerve compression model, the contralateral and ipsilateral DRG tissues were removed. Total proteins were extracted from tissues using radioimmunoprecipitation assay lysis buffer containing $1 \%$ phenylmethanesulfonyl fluoride and phosphatase inhibitor (Wuhan Boster Biological Technology, Ltd.), and lysed on ice for $30 \mathrm{~min}$. After centrifugation at $12,000 \mathrm{x}$ g for $10 \mathrm{~min}$ at $4^{\circ} \mathrm{C}$, the supernatant was collected and stored at $-80^{\circ} \mathrm{C}$ until used. The protein concentration was measured using a bicinchoninic acid protein assay kit (Beyotime Institute of Biotechnology) in accordance with the manufacturer's protocol. The samples were resolved using either 10\% SDS-PAGE (for CTGF, collagen I and GAPDH; Wuhan Boster Biological Technology, 
Ltd.) or $15 \%$ (for TGF- $\beta 1$ ) SDS-PAGE, followed by transfer onto a polyvinylidene fluoride membrane (eBioscience, Inc., San Diego, CA, USA), and blocking with 5\% w/v non-fat dry milk. The membranes were then incubated with primary antibodies at $4^{\circ} \mathrm{C}$ overnight as follows: Anti-TGF- $\beta 1$ (1:500 dilution), anti-CTGF (1:1,000 dilution), anti-collagen I (1:5,000 dilution), anti-GAPDH (1:10,000 dilution), anti-p-ERK (1:1,000 dilution), anti-p-p38 (1:1,000 dilution) and anti-p-JNK (1:1,000 dilution). Following washing, the membranes were incubated with HRP-labeled secondary antibodies (1:10,000 dilution) at $4^{\circ} \mathrm{C}$ overnight, then washed with TBS-T (0.1\% Tween- 20$)$.

The resulting immunobands were visualized using an Enhanced Chemiluminescence kit (EMD Millipore, Billerica, MA, USA), and the densitometric values were used for statistical analysis. The housekeeping protein, GAPDH, was used as an internal control.

Statistical analyses. The data were analyzed using SPSS 17.0 software (SPSS, Inc., Chicago, IL, USA). Data are presented as the mean \pm standard deviation. $\mathrm{P}<0.05$ was considered to indicate a statistically significant difference. Statistical significance between two groups was determined using Student's $t$-test.

\section{Results}

Chronic sciatic nerve compression-induced pathology. The present study examined the pathological changes in the rat chronic sciatic nerve compression model. At 3 weeks and 8 weeks post-surgery, Masson's trichrome staining showed normal gastrocnemius muscle fiber structure and collagen fiber morphology on the contralateral side. However, at 3 weeks, collagen fiber accumulation was detected on the ipsilateral side and, at 8 weeks, excessive collagen fiber formation and muscle atrophy were observed (Fig. 1A).

The chronic sciatic nerve compression in the model group was associated with lower levels of gastrocnemius muscle fiber CSA ag 3 weeks post-surgery, which was significantly lower at 8 weeks, compared with the contralateral side (Fig. 1B). In the model rats, the percentage of gastrocnemius muscle fiber CVF was significantly higher on the injured side, compared with the contralateral side at 3 weeks, and this difference was more marked at 8 weeks (Fig. 1C). In the subsequent experiments, the molecular and cellular changes in the DRG neurons 3 weeks following nerve compression.

Retrograde fluorescent tracing of DRG neurons. In the present study, the FR-labeled neurons exhibited red fluorescence, predominantly in the cytoplasm, and the FG-labeled neurons appeared bright blue under the fluorescent microscope (Fig. 2). The majority of the DRG neurons were stained with FG, and no significant difference was detected in the number of FG-labeled neurons between the sham control group and the group of rats with compression on the injured side $(\mathrm{P}>0.05)$.

By contrast, the number of FR-labeled DRG neurons was significantly higher in the rats with chronic sciatic nerve compression, compared with the sham control group $(\mathrm{P}<0.05)$. Few neurons were stained with both FR and FG. No significant differences were observed in the total number of neurons labeled with FR, FG or the two together, between the two groups $(\mathrm{P}>0.05)$.
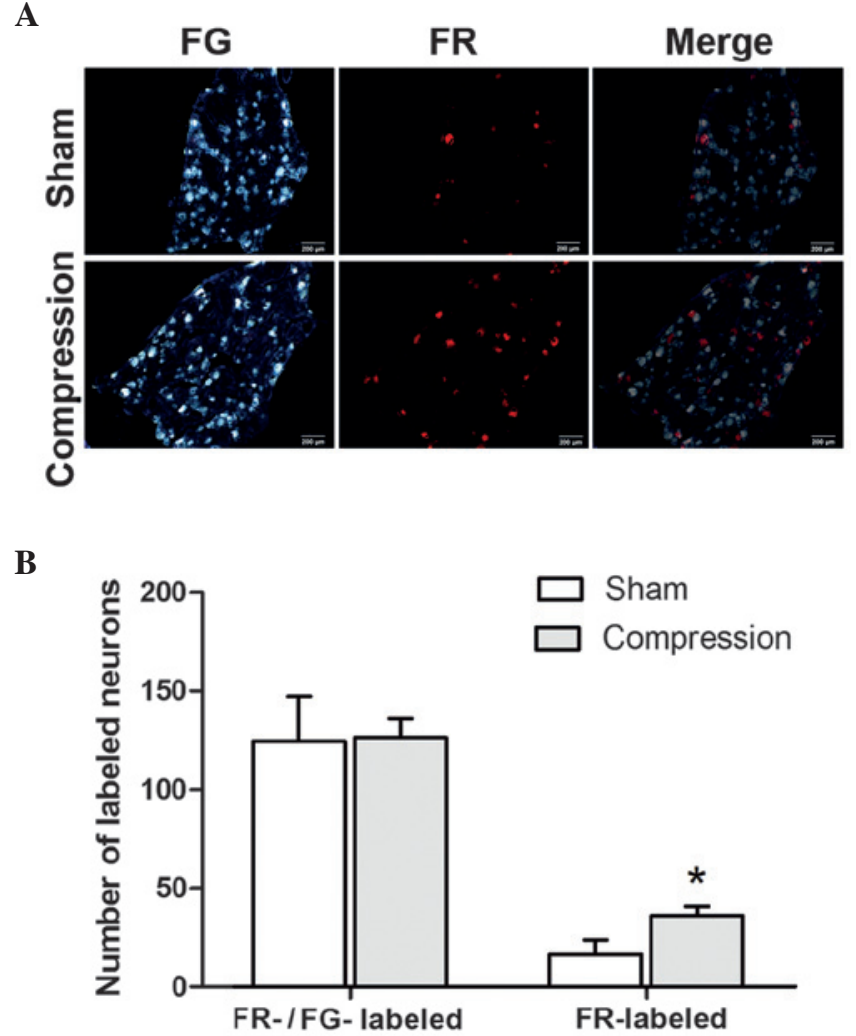

Figure 2. Retrograde fluorescent tracing of DRG neurons. Tissues from rats in the sham control and chronic sciatic nerve compression groups were labeled with FR and FG. (A) FR (red) and FG (blue) labeling of DRG neurons. Scale bar $=200 \mu \mathrm{m}$. (B) Numbers of FR- and FG-positive neurons per field. Data are presented as the mean \pm standard deviation. ${ }^{*} \mathrm{P}<0.05$, compared with the sham control group. DRG, dorsal root ganglion; FR, Fluoro Ruby; FG, Fluoro Gold.

Expression of TGF- $\beta 1, C T G F$ and collagen type I in injured $D R G$ neurons and surrounding tissues. In the model rats, the present study found that the mRNA expression levels of TGF- $\beta 1$, CTGF and collagen type I were significantly higher in the ipsilateral DRG neurons, compared with the contralateral DRG neurons $(\mathrm{P}<0.05$; Fig. 3A). Consistent with this, the protein levels of TGF- $\beta 1$, CTGF and collagen type I were also higher in the ipsilateral DRG neurons and surrounding tissues, compared with the those on the contralateral side $(\mathrm{P}<0.05$; Fig. 3B and C).

Immunohistochemical analysis showed low levels of expression of TGF- $\beta 1$, CTGF and collagen type I in the contralateral DRG neurons and surrounding tissues, whereas their levels of expression were higher in the ipsilateral DRG neurons and surrounding tissues (Fig. 3D). Additionally, TGF- $\beta 1$ and CTGF were predominantly distributed in the cytoplasm and axons of the DRG neurons, and collagen type I was expressed in the tissues surrounding the neurons.

Involvement of the MAPK signaling pathway in chronic sciatic nerve compression-induced DRG injury. Activation of the three major MAPK members, ERK1/2, JNK and p38 MAPK is known to modulate the pathogenesis of fibrosis in multiple organs (22-24). In the present study, immunoblotting revealed that, in the model rats, the protein levels of p-ERK1/2 were higher in the ipsilateral DRGs following chronic nerve 
A

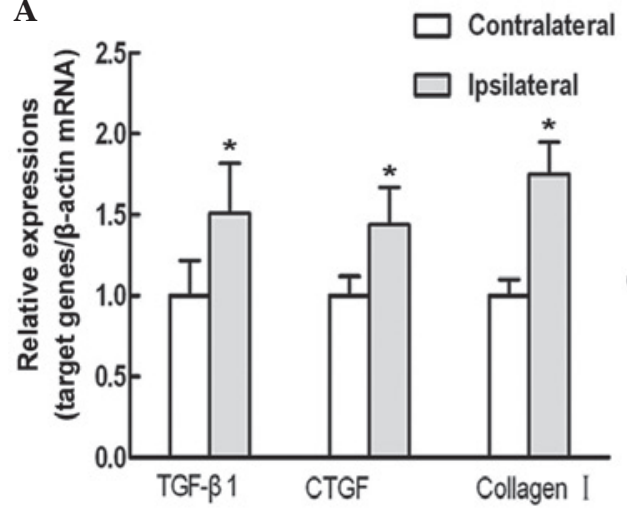

B

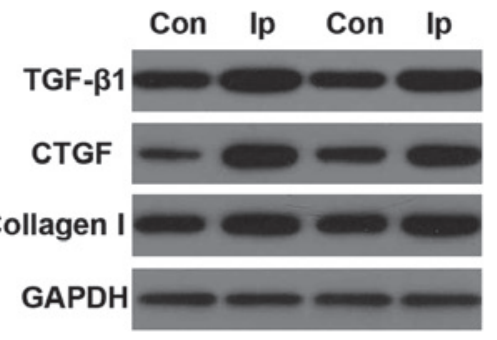

C

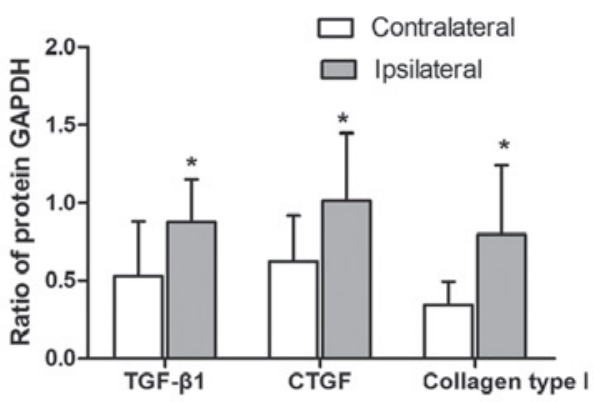

D

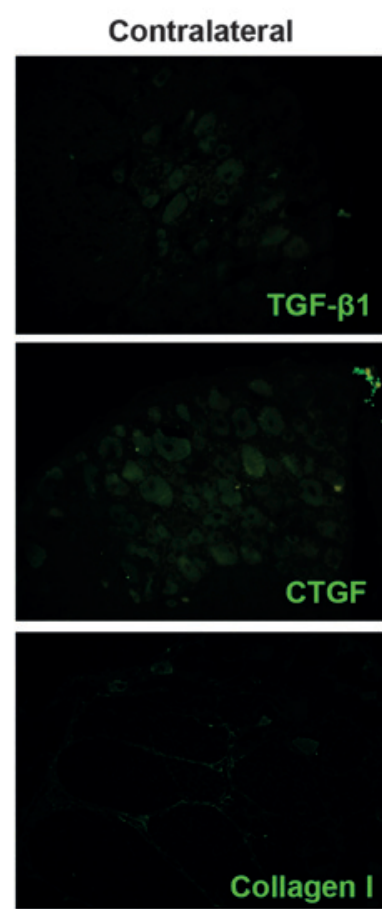

Contralateral
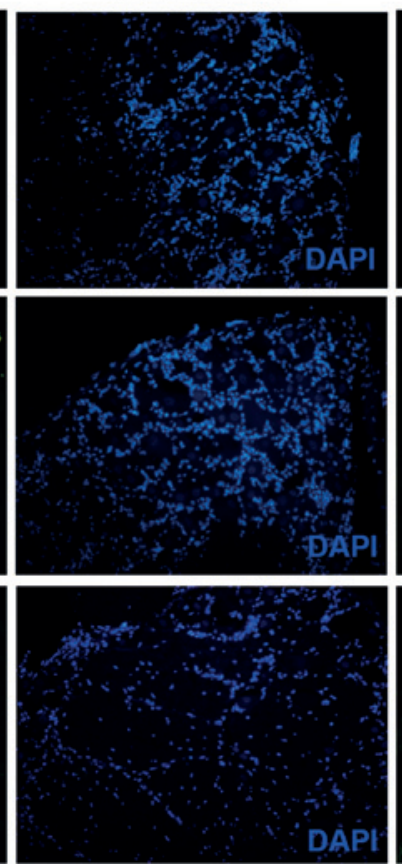

Ipsilateral
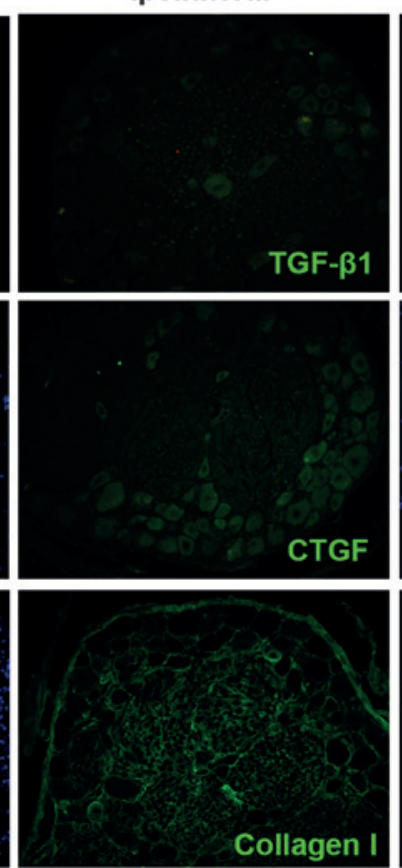

Ipsilateral
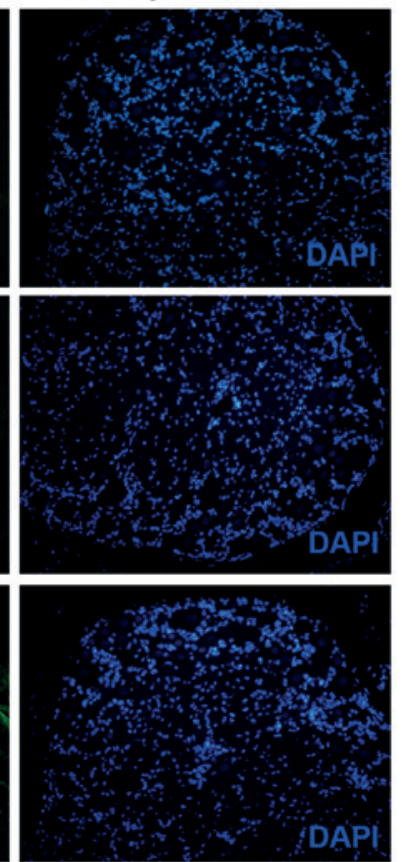

Figure 3. mRNA and protein levels of TGF- $\beta 1$, CTGF and collagen type I in DRG neurons. (A) mRNA levels of target genes in contralateral and ipsilateral DRG neurons were examined using reverse transcription-quantitative polymerase chain reaction 3 weeks following surgery (n=4). (B) Protein levels of target genes were evaluated using western blotting. Representative results are shown. (C) Relative protein levels were calculated ( $\mathrm{n}=3$ ). (D) Levels and distribution of target proteins were examined using immunohistochemistry. Nuclei were counterstained with DAPI ( $\mathrm{n}=10)$. Magnification, $\mathrm{x} 200$. Data are presented as the mean \pm standard deviation. ${ }^{*} \mathrm{P}<0.05$, compared with the contralateral DRG. TGF- $\beta 1$, transforming growth factor- $\beta 1$; CTGF, connective tissue growth factor; GAPDH, glyceraldehyde 3-phosphate dehydrogenase; DRG, dorsal root ganglion; Con, contralateral; Ips, ipsilateral.

$\mathbf{A}$

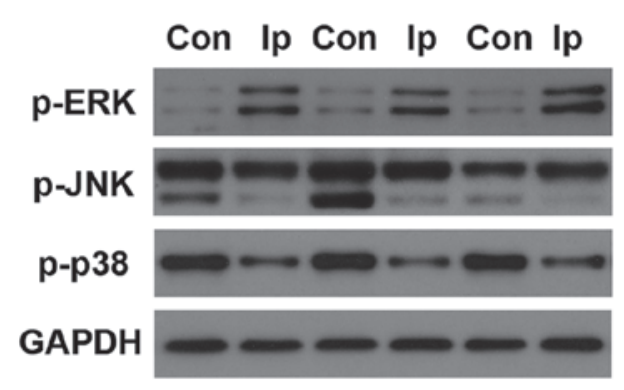

B

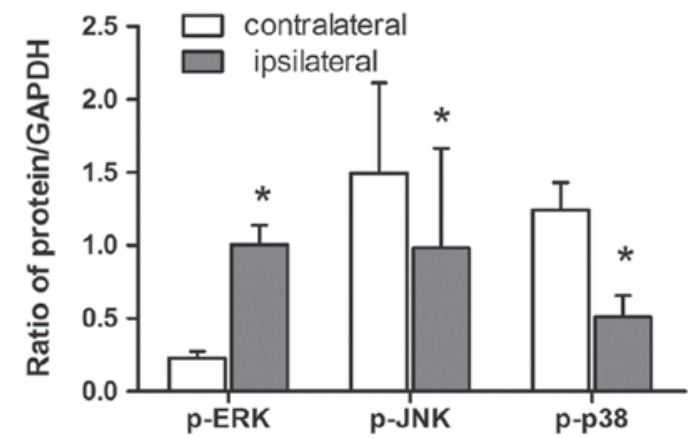

Figure 4. Involvement of the mitogen-activated protein kinase signaling pathway in chronic sciatic nerve compression-induced DRG injury. (A) Levels of p-ERK, p-p38 and p-JNK in the ipsilateral and contralateral DRGs following chronic nerve compression. Representative results are shown. (B) Relative protein expression levels were calculated. Data are presented as the mean \pm standard deviation. ${ }^{*} \mathrm{P}<0.05$ compared with contralateral DRG $(\mathrm{n}=3)$. ERK, extracellular signal-regulated kinase; JNK, c-Jun N-terminal kinase; p-, phosphorylated; GAPDH, glyceraldehyde 3-phosphate dehydrogenase; DRG, dorsal root ganglion;Con, contralateral; Ips, ipsilateral. 
compression, compared with the levels in the contralateral DRGs ( $\mathrm{P}<0.05$; Fig. 4). By contrast, chronic nerve compression was associated with significantly lower levels of p-JNK and p-p38 in the DRGs $(\mathrm{P}<0.05)$.

\section{Discussion}

In the present study, a rat model was used to investigate the changes associated with chronic sciatic nerve compression in neurons of the DRG. The evidence suggested that chronic sciatic nerve compression led to higher levels of TGF- $\beta 1$, CTGF and collagen type I, which may have been responsible for inducing fibrosis in the tissues close to the lesions. Furthermore, differences associated with chronic sciatic nerve compression in the levels of the major MAPK members, ERK1/2, JNK and p38 MAPK, suggested the involvement of the MAPK signaling pathway in the pathogenesis of nerve compression injury.

Chronic sciatic nerve compression is characterized by early damage to the blood-nerve barrier, which leads to swelling and fibrosis, Schwann cell proliferation, local demyelination, and Wallerian degeneration of the nerve (5). To understand the precise pathological changes, as well as the molecular and cellular changes of DRG neurons following chronic nerve compression, the present study established a rat model of chronic sciatic nerve compression, as previously described (19).

It has been demonstrated that demyelinated axons can be differentially labeled with FR, and retrograde axonal transport of this fluorescent tracer has also been shown, in which FR evident in axonal fibers at the site of the compressed nerve can be used to distinguish damaged neurons from healthy neurons (9). In accordance with this observation, the present study detected a significantly higher number of FR-labeled neurons in the model of nerve compression, compared with the contralateral control. By contrast, the uptake of FG was demonstrated in all neurons, whether injured or normal, which is consistent with a previous report (25). In addition, moderate pathological changes were observed 3 weeks following sciatic nerve compression, and nerve injury and muscle atrophy were evident at 8 weeks.

A previous study examined injured nerves and surrounding muscles using magnetic resonance imaging, and found structural abnormalities (26). In addition, enhanced FDG uptake was detected in these area using (18F)-2-fluoro-2-deoxy-d-glucose positron emission tomography (26). Therefore, the present study investigated the molecular mechanism involved in chronic sciatic nerve compression-induced DRG damage and muscle fibrosis 3 weeks following surgery.

Collagen type I is an important component of mammalian extracellular matrix (27). During injury, fibroblasts migrate into the damaged area, proliferate and synthesize extracellular matrix components, particularly collagen type I (28). Collagen deposition is essential in the regulation of Schwann cell function following nerve injury (29), and the overproduction of collagen type I may contribute to the pathogenesis of fibrosis (12). In the present study, higher levels of collagen type I were observed surrounding the ipsilateral DRG, compared with the contralateral DRG. This suggested that proliferated fibroblasts may generate collagen type I, and that extracellular matrix accumulation and tissue fibrosis may occur in damaged nerve tissues.
In pathological conditions, TGF- $\beta 1$ and CTGF, which are crucial mediators of tissue fibrosis, lead to the accumulation of extracellular matrix (30). TGF- $\beta 1$ mediates tissue fibrosis by inducing the activation of CTGF $(30,31)$, which upregulates the expression of CTGF by activating small mothers against decapentaplegic. The activated CTGF subsequently mediates downstream effectors, which collectively contribute to fibrosis $(31,32)$. CTGF is key in the development of tissue fibrosis by inducing collagen type $\mathrm{I}$ in the surrounding tissues $(33,34)$. Reduced liver fibrosis has been observed in rats lacking CTGF expression (35). Therefore, TGF- $\beta 1$ and CTGF are recognized as multifaceted regulatory proteins in fibrosis (36). In the present study, the levels of TGF- $\beta 1$ and CTGF were higher in ipsilateral DRG neurons, compared with contralateral DRG neurons. It is possible that chronic sciatic nerve compression triggered the generation of TGF- $\beta 1$, and the consequent activation and release of CTGF in the DRG neurons; and this increased expression of TGF- $\beta 1$ and CTGF led to the production of excessive collagen type I in the surrounding tissues, which finally contributed to fibrosis. It is also possible that the TGF- $\beta 1$ released from DRG neurons (37) had a direct connection with the paracrine CTGF signal. The cysteine-rich domain of CTGF directly binds to and induces the activation of TGF- $\beta 1$ (38), and activated TGF- $\beta 1$ binds to its receptor on fibroblasts, glia or Schwann cells to induce the generation of collagen type I $(39,40)$. However, the precise mechanism requires further clarification.

The ERK1/2, JNK and p38 MAPK pathway transduction signals are known to be involved in various intracellular signaling pathways, which control a wide spectrum of cellular processes, including fibrosis $(41,42)$. The activation of MAPK signals, mediated by TGF- $\beta 1$, contribute to the deposition of extracellular matrix and fibrosis (30). In addition, activation of the ERK signaling molecule has been shown to accompany fibroblast activation and collagen synthesis (43). In the present study, ERK1/2 was activated in the ipsilateral DRG neurons, indicated by higher levels of p-ERK1/2. Consistent with the observations in the present study of increased activation of ERK1/2 in compressed DRGs, it has been reported that p-ERK1/2 induces neuropathic pain in DRG neurons, and gabapentin has an analgesic effect by inhibiting the activation of ERK1/2 activation (44). However, the effects of the activation of ERK $1 / 2$ and the downstream effectors of ERK1/2 remain to be elucidated. Although the activation of JNK and p38 has been detected in the development of neuropathic pain in cultured DRG neurons (45), chronic nerve compression in the present study was associated with significantly lower levels of p-JNK and p-p38 in the DRGs. This discrepancy may be due to differences between in vitro and in vivo conditions. The evidence suggested that MAPK members may be involved in chronic sciatic nerve compression-induced DRG damage, and future investigations aim to investigate the underlying mechanism involved.

In conclusion, the present study demonstrated that chronic sciatic nerve compression induced DRG impairment. It is possible that increased expression levels of TGF- $\beta 1$ and CTGF in the DRG neurons, and elevated expression of collagen type I in the surrounding tissues, may contribute to the fibrosis induced by chronic sciatic nerve compression. 


\section{Acknowledgements}

This study was supported by the National Natural Science Foundation of China (grant nos. 81271967, 81471270 and 81252899).

\section{References}

1. Pelletier J, Fromy B, Morel G, Roquelaure Y, Saumet JL and Sigaudo-Roussel D: Chronic sciatic nerve injury impairs the local cutaneous neurovascular interaction in rats. Pain 153: 149-157, 2012.

2. Berger JV, Knaepen L, Janssen SP, Jaken RJ, Marcus MA, Joosten EA and Deumens R: Cellular and molecular insights into neuropathy-induced pain hypersensitivity for mechanism-based treatment approaches. Brain Res Rev 67: 282-310, 2011.

3. Pham K and Gupta R: Understanding the mechanisms of entrapment neuropathies. Review article. Neurosurg Focus 26: E7, 2009.

4. Gupta R, Rowshan K, Chao T, Mozaffar T and Steward O: Chronic nerve compression induces local demyelination and remyelination in a rat model of carpal tunnel syndrome. Exp Neurol 187: 500-508, 2004.

5. Mackinnon SE: Pathophysiology of nerve compression. Hand Clin 18: 231-241, 2002.

6. Mackinnon SE, Dellon AL, Hudson AR and Hunder DA: Chronic human nerve compression - a histological assessment. Neuropathol Appl Neurobiol 12: 547-565, 1986.

7. Prinz RA, Nakamura-Pereira M, De-Ary-Pires B, Fernandes D, Fabião-Gomes BD, Martinez AM, de Ary-Pires R, Pires-Neto MA: Axonal and extracellular matrix responses to experimental chronic nerve entrapment. Brain Res 1044: 164-175, 2005.

8. Khoa Pham, Nima Nassiri, Ranjan Gupta. c-Jun, krox-20, and integrin $\beta 4$ expression following chronic nerve compression injury. Neurosci Lett 465: 194-198, 2009.

9. Chao T, Pham K, Steward O and Gupta R: Chronic nerve compression injury induces a phenotypic switch of neurons within the dorsal root ganglia. J Comp Neurol 506: 180-193, 2008.

10. Zhang Y, Wang YH, Zhang XH, Ge HY, Arendt-Nielsen L, Shao JM and Yue SW: Proteomic analysis of differential proteins related to the neuropathic pain and neuroprotection in the dorsal root ganglion following its chronic compression in rats. Exp Brain Res 189: 199-209, 2008

11. Dubovy P, Brazda V, Klusakova I and Hradilova-Svizenska I: Bilateral elevation of interleukin- 6 protein and mRNA in both lumbar and cervical dorsal rootganglia following unilateral chronic compression injury of the sciatic nerve. J Neuroinflammation 10 : 55, 2013.

12. Ponticos M, Papaioannou I, Xu S, et al: The failure to degrade JunB contributes to Collagen type I over-production and dermal fibrosis in Scleroderma. Arthritis Rheumatol 1: 242-253, 2014.

13. Zhu Y, Colak T, Shenoy M, Liu L, Mehta K, Pai R, Zou B, Xie XS and Pasricha PJ: Transforming growth factor beta induces sensory neuronal hyperexcitability and contributes to pancreatic pain and hyperalgesia in rats with chronic pancreatitis. Mol Pain 8: 65, 2012.

14. Fujii M, Nakanishi H, Toyoda T, Tanaka I, Kondo Y, Osada H and Sekido Y: Convergent signaling in the regulation of connective tissue growth factor in malignant mesothelioma: TGF $\beta$ signaling and defects in the Hippo signaling cascade. Cell Cycle 11: 3373-3379, 2012.

15. Peng B, Chen J, Kuang Z, Li D, Pang X and Zhang X: Expression and role of connective tissue growth factor in painful disc fibrosis and degeneration. Spine (Phila Pa 1976) 34: E178-E182, 2009.

16. Yang Y, Wu H, Yan JQ, Song ZB and Guo QL: Tumor necrosis factor- $\alpha$ inhibits angiotensin II receptor type 1 expression in dorsal root ganglion neurons via $\beta$-catenin signaling. Neuroscience 248: 383-391, 2013.

17. Muralidharan A, Wyse BD and Smith MT: Analgesic efficacy and mode of action of a selective small molecule angiotensin II type 2 receptor antagonist in a rat model of prostate cancer-induced bone pain. Pain Med 15: 93-110, 2014.

18. Obata K and Noguchi K: MAPK activation in nociceptive neurons and pain hypersensitivity. Life Sci 74: 2643-2653, 2004.

19. O'Brien JP, Mackinnon SE, MacLean AR, Hudson AR, Dellon AL and Hunter DA: A model of chronic nerve compression in the rat. Ann Plast Surg 19: 430-435, 1987.
20. Zele T, Sketelj J and Bajrovic FF: Efficacy of fluorescent tracers in retrograde labeling of cutaneous afferent neurons in the rat. J Neurosci Methods 191: 208-214, 2010.

21. Livak KJ and Schmittgen TD: Analysis of relative gene expression data using real-time quantitative PCR and the $2^{-\Delta \Delta C t}$ method. Methods 25: 402-408, 2001.

22. Madala SK, Schmidt S, Davidson C, Ikegami M, Wert S and Hardie WD: MEK-ERK pathway modulation ameliorates pulmonary fibrosis associated with epidermal growth factor receptor activation. Am J Respir Cell Mol Biol 46: 380-388, 2012.

23. Gao X, Wu G, Gu X, Fu L and Mei C: Kruppel-like factor 15 modulates renal interstitial fibrosis by ERK/MAPK and JNK/MAPK pathways regulation. Kidney Blood Press Res 37:631-640, 2013

24. Ma FY, Tesch GH and Nikolic-Paterson DJ: ASK1/p38 signaling in renal tubular epithelial cells promotes renal fibrosis in the mouse obstructed kidney. Am J Physiol Renal Physiol 307: F1263-F1273, 2014.

25. Kawarai Y, Suzuki M, Yoshino K, Inoue G, Orita S, Yamauchi K, Aoki Y, Ishikawa T, Miyagi M, Kamoda H, et al: Transient receptor potential vanilloid 1-immunoreactive innervation increases in fractured rat femur. Yonsei Med J 55: 185-190, 2014.

26. Lasko L, Huang X, Voorbach MJ, Lewis LG, Stavropoulos J, Carriker J, Seifert TR, Baker SJ and Upadhyay J: Multimodal assessment of nervous and immune system responses following sciatic nerve injury. Pain 154: 2782-2793, 2013.

27. Jones CA, Liang L, Lin D, Jiao Y and Sun B: The spatial-temporal characteristics of type I collagen-based extracellular matrix. Soft Matter 10: 8855-8863, 2014.

28. Cheret J, Lebonvallet N, Buhe V, Carre JL, Misery L and Le Gall-Ianotto C: Influence of sensory neuropeptides on human cutaneous wound healing process. J Dermatol Sci 74: 193-203, 2014.

29. Koopmans G, Hasse B and Sinis N: Chapter 19: The role of collagen in peripheral nerve repair. Int Rev Neurobiol 87: 363-379, 2009.

30. Ihn H: Pathogenesis of fibrosis: Role of TGF-beta and CTGF. Curr Opin Rheumatol 14: 681-685, 2002.

31. Leask A, Holmes A, Black CM and Abraham DJ: Connective tissue growth factor gene regulation. Requirements for its induction by transforming growth factor-beta 2 in fibroblasts. J Biol Chem 278: 13008-13015, 2003.

32. Igarashi A, Okochi H, Bradham DM and Grotendorst GR: Regulation of connective tissue growth factor gene expression in human skin fibroblasts and during wound repair. Mol Biol Cell 4: 637-645, 1993

33. Boerma M, Wang J, Sridharan V,Herbert JM and Hauer-Jensen M: Pharmacological induction of transforming growth factor-beta1 in rat models enhances radiation injury in the intestine and the heart. PLoS One 8: e70479, 2013.

34. Sonnylal S, Xu S, Jones H, Tam A, Sreeram VR, Ponticos M, Norman J, Agrawal P, Abraham D and De Crombrugghe B: Connective tissue growth factor causes EMT-like cell fate changes in vivo and in vitro. J Cell Sci 126: 2164-2175, 2013.

35. George J and Tsutsumi M: siRNA-mediated knockdown of connective tissue growth factor prevents $\mathrm{N}$-nitrosodimethylami ne-induced hepatic fibrosis in rats. Gene Ther 14: 790-803, 2007.

36. Gressner OA and Gressner AM: Connective tissue growth factor: A fibrogenic master switch in fibrotic liver diseases. Liver Int 28: 1065-1079, 2008.

37. Stark B, Carlstedt T and Risling M: Distribution of TGF-beta, the TGF-beta type I receptor and the R-II receptor in peripheral nerves and mechanoreceptors; observations on changes after traumatic injury. Brain Res 913: 47-56, 2001.

38. Abreu JG, Ketpura NI, Reversade B and De Robertis EM: Connective-tissue growth factor (CTGF) modulates cell signalling by BMP and TGF-beta. Nat Cell Biol 4: 599-604, 2002.

39. Liu Y, Liu Z, Liu X, Luo B, Xiong J, Gan W, Jiang M, Zhang Z, Schluesener HJ and Zhang Z: Accumulation of connective tissue growth factor+ cells during the early phase of rat traumatic brain injury. Diagn Pathol 9: 141, 2014.

40. Petito RB, Amadeu TP, Pascarelli BM, Jardim MR, Vital RT, Antunes SL and Sarno EN: Transforming growth factor- $\beta 1$ may be a key mediator of the fibrogenic properties of neural cells in leprosy. J Neuropathol Exp Neurol 72: 351-366, 2013.

41. Peti W and Page R: Molecular basis of MAP kinase regulation. Protein Sci 22: 1698-1710, 2013. 
42. Okada Y, Shirai K, Reinach PS, Kitano-Izutani A, Miyajima M, Flanders KC, Jester JV, Tominaga M and Saika S: TRPA1 is required for TGF- $\beta$ signaling and its loss blocks inflammatory fibrosis in mouse corneal stroma. Lab Invest 94: 1030-1041, 2014.

43. Zhang YP, Wang WL, Liu J, Li WB, Bai LL, Yuan YD and Song SX: Plasminogen activator inhibitor-1 promotes the proliferation and inhibits the apoptosis of pulmonary fibroblasts by $\mathrm{Ca}$ (2+) signaling. Thromb Res 131: 64-71, 2013.
44. Zhang JL, Yang JP, Zhang JR, Li RQ, Wang J, Jan JJ and Zhuang Q: Gabapentin reduces allodynia and hyperalgesia in painful diabetic neuropathy rats by decreasing expression level of Nav1.7 and p-ERK1/2 in DRG neurons. Brain Res 1493: 13-18, 2013.

45. Zang Y, Xin WJ, Pang RP, Li YY and Liu XG: Upregulation of Nav1.3 Channel Induced by rrTNF in Cultured Adult Rat DRG Neurons via p38 MAPK and JNK Pathways. Chin J Physiol 54: 241-246, 2011. 